\title{
Linear Dynamically Varying Linear Quadratic Control of Systems with Complicated Dynamics
}

\author{
S. Bohacek and E. Jonckheere \\ Dept. of Mathematics and Dept. of Electrical Engineering-Systems \\ University of Southern California \\ Los Angeles, CA 90089-2563 \\ bohacek@usc.edu,jonckhee@eudoxus.usc.edu
}

Fax: (213) 740-4449

\begin{abstract}
Linear quadratic controllers for dynamical systems with complicated dynamics are presented. Systems with complicated dynamics are those that run over compact sets and have such features as nontrivial recurrence, periodic and aperiodic orbits. The controllers are based on modeling the nonlinear dynamical systems as linear dynamically varying systems. Necessary and sufficient conditions for the existence of such controllers are linked to the existence of a bounded solution to a functional algebraic Riccati equation. Several methods to solve the functional Riccati equation are presented. Finally, an example of controlling the Hénon map is presented.
\end{abstract}

\section{Introduction}

This paper presents a control strategy for systems with complicated dynamics. By complicated dynam$i c s$, we mean a discrete-time, nonlinear dynamical system running over a compact set $\Theta$ which exhibits nontrivial recurrence. By control, we mean tracking one of the natural periodic or aperiodic orbits of the dynamics.

A specific feature of tracking a natural periodic or aperiodic motion is that the objective can be achieved with low cost control. Therefore, the control is taken to be a very small perturbation of the parameters of the nominal dynamics. More specifcally, the nominal and perturbed dynamics are, respectively,

$$
\begin{array}{rlrl}
\theta(k+1) & =f(\theta(k)), & & \theta(0)=\theta_{0} \in \Theta, \\
\varphi(k+1) & =f(\varphi(k), u(k)), & \varphi(0)=\varphi_{0} \in \Theta,
\end{array}
$$

where $f: \mathbb{R}^{n} \times \mathbb{R}^{m} \rightarrow \mathbb{R}^{n}, f \in C^{1}\left(\mathbb{R}^{n} \times \mathbb{R}^{m}, \mathbb{R}^{n}\right)$, $\Theta \subset \mathbb{R}^{n}$ is compact, and $f(\Theta, 0) \subseteq \Theta$. Thus $\{\theta(k): k \geq 0\}$ is the desired trajectory and $\varphi(k)$ is the state of the system under control. The objective is to find a control $u$ such that $\lim _{k \rightarrow \infty}\|\varphi(k)-\theta(k)\|=0$.

This paper develops a controller of the form $u(k)=$
$F_{\theta(k)}(\varphi(k)-\theta(k))$, where the feedback $F_{\theta}$ is designed from a linearized approximation of $f(\varphi, u)$ around $(\theta, 0)$, and the gain $F_{\theta}$ is "scheduled" so as to follow the desired trajectory. The controller $F_{\theta}$ exists if a solution to a functional algebraic Riccati equation (FARE) exists. The mathematical difficulty with this functional equation is to prove that the relevant solution is continuous, in which case $F_{\theta}$ is continuous.

Typically, there is no closed form solution to the FARE. However, dynamical systems on compact sets, subject to some mild additional conditions, are known to have such ergodic properties as recurrence, which can be put into use to construct an approximate solution of arbitrary accuracy.

The proofs of the results in this paper are provided in [1], [3] and [4].

\section{Controlling Dynamical Systems with Linear Dynamically Varying Control}

Consider a slightly generalized version of the problem posed in Section 1:

$$
\begin{gathered}
\theta(k+1)=f(\theta(k), 0), \text { with } \theta(0)=\theta_{o}, \\
\varphi(k+1)=f(\varphi(k), u(k)), \text { with } \varphi(0)=\varphi_{0} .
\end{gathered}
$$

The popular problem of getting $\varphi$ to follow a periodic orbit fits into this framework by setting $\theta(0) \in P(f)$, where $P(f)$ is the set of periodic points. However, if the objective is for $\varphi$ to follow an aperiodic orbit, then $\theta(0) \in N W(f) \backslash P(f)$, where $N W(f)$ is the set of nonwandering points.

The targeting problem also fits within this framework. Initially, the state is forced to follow a targeting trajectory that originates near the current state, and ends near a point of the desired orbit. If the system is transitive, such a targeting trajectory exists. Once the state has reached the end of the targeting trajectory, a second control is applied to force the system to follow the desired orbit. This switching 
of trajectories is accomplished by allowing for occasional small jumps in $\theta(k)$.

Now we define the tracking error $x(k)=\varphi(k)$ $\theta(k)$. The linear approximation of $f(\varphi(k), u)$ around $\varphi(k)=\theta(k)$ and $u(k)=0$ yields

$$
\begin{aligned}
x(k+1)= & A_{\theta(k)} x(k)+B_{\theta(k)} u(k) \\
& +\eta(x(k), u(k), \theta(k))
\end{aligned}
$$

where $A_{\theta}=\frac{\partial f}{\partial x}(\theta, 0), \quad B_{\theta}=\frac{\partial f}{\partial u}(\theta, 0)$ and

$$
\begin{aligned}
\eta(x(k), u(k), \theta(k))= & \eta_{x}(x(k), u(k),(\theta)) x(k)(4) \\
& +\eta_{u}(x(k), u(k), \theta(k)) u(k)
\end{aligned}
$$

Furthermore, $\left\|\eta_{x}(x, u, \theta)\right\|$ and $\left\|\eta_{u}(x, u, \theta)\right\|$ can be made as small as necessary by limiting the size of $u$ and $x$.

If $u$ and $x$ are small, then we can approximate the error dynamics as

$$
\begin{aligned}
& x(k+1)=A_{\theta(k)} x(k)+B_{\theta(k)} u(k) \\
& \dot{\theta}(k+1)=f(\theta(k))
\end{aligned}
$$

This is a linear system with coefficient matrices $A$ and $B$ that vary as $\theta(k)$ varies. Since $\theta(k)$ varies according to equation (1), system (5) is a Linear $D y$ namically Varying ( $L D V$ ) system.

Our objective is to not only force the tracking error to go to zero asymptotically, but go to zero uniformly exponentially fast. We distinguish the various forms of stability as follows.

Definition 1 The linear dynamically varying system (5) is uniformly exponentially stable if for $u(k)=0$, there exists an $0<\alpha<1$ and $a \beta<\infty$ such that for all $\theta(0) \in \Theta$

$$
\|x(k)\| \leq \beta \alpha^{k}\|x(0)\| .
$$

System (5) is exponentially stable, if for $u(k)=0$ and for each $\theta(0) \in \Theta$, there exists an $0<\alpha(\theta(0))<$ 1 and $a \beta(\theta(0))<\infty$ such that for all $x(j)$ and $j \leq k$

$$
\|x(k)\| \leq \beta(\theta(0)) \alpha(\theta(0))^{k-j}\|x(j)\| .
$$

It was shown in [1] that in the case of continuous LDV systems, exponential and uniform exponential stability are equivalent. Since uniformly exponentially stable systems are inherently more robust than exponentially stable systems, it is preferable to remain within the confines of continuous LDV systems. Thus when synthesizing a feedback for controlling a continuous LDV system, it is important to ensure that the feedback is not only stabilizing, but also continuous. However, to maintain generality, an LDV system is considered stabilizable if there exists an exponentially stabilizing feedback, that is:

Definition 2 System (5) is stabilizable if there exists $a$, not necessarily continuous, function $F: \mathbb{N} \times$
$\Theta \rightarrow \mathbb{R}^{m \times n}$ such that for all $\theta(0) \in \Theta$ and for all $k$, $\|F(k, \theta(0))\| \leq \bar{F}(\theta(0))<\infty$, and

$$
\begin{aligned}
x(k+1) & =\left(A_{\theta(k)}+B_{\theta(k)} F(k, \theta(0))\right) x(k) \\
\theta(k) & =f^{k}(\theta(0))
\end{aligned}
$$

is exponentially stable.

Our main result can now be formulated:

Theorem 1 Assume that system (5) is stabilizable. Then there exists a unique, bounded solution $X$ : $\Theta \rightarrow \mathbb{R}^{n \times n}$ such that $X_{\theta}=X_{\theta}^{\prime} \geq 0$,

$X_{\theta}=A_{\theta}^{\prime} X_{f(\theta)} A_{\theta}+I$

$$
-A_{\theta}^{\prime} X_{f(\theta)} B_{\theta}\left(I+B_{\theta}^{\prime} X_{f(\theta)} B_{\theta}\right)^{-1} B_{\theta}^{\prime} X_{f(\theta)} A_{\theta}
$$

and the feedback

$$
\begin{aligned}
u(k)= & -\left(I+B_{\theta(k)}^{\prime} X_{f(\theta(k))} B_{\theta(k)}\right)^{-1} \\
& \times B_{\theta(k)}^{\prime} X_{f(\theta(k))} A_{\theta(k)} x(k)
\end{aligned}
$$

uniformly exponentially stabilizes system (5). For $\|x(0)\|<\infty$, this feedback minimizes $\sum_{k=0}^{\infty}\|x(k)\|^{2}+$ $\|u(k)\|^{2}$ and $X$ is a uniformly continuous function.

Conversely, if there exists a bounded solution, $X$, to equation (6) such that $X_{\theta}=X_{\theta}^{\prime} \geq 0$, then system (5) is stabilizable and $X$ is continuous. In this case, if $X(k, N, \theta)$ solves the finite horizon Riccati equation, i.e.

$$
\begin{aligned}
& X(k, N, \theta) \\
= & A_{f^{k}(\theta)}^{\prime} X(k+1, N, \theta) A_{f^{k}(\theta)} \\
& +I-A_{f^{k}(\theta)}^{\prime} X(k+1, N, \theta) B_{f^{k}(\theta)} \\
& \times\left(I+B_{f^{k}(\theta)}^{\prime} X(k+1, N, \theta) B_{f^{k}(\theta)}\right)^{-1} \\
& \times B_{f^{k}(\theta)}^{\prime} X(k+1, N, \theta) A_{f^{k}(\theta)}
\end{aligned}
$$

with

$$
X(N, N, \theta)=I,
$$

then $X(0, N, \theta) \rightarrow X_{\theta}$ uniformly in $\theta$.

Remark 1 Stabilizability is a rather weak assumption. Indeed, stabilizability merely assumes that every trajectory is stabilizable. Of course, one could not hope to stabilize every trajectory if some trajectories are not stabilizable. Given this obviously necessary condition, it is interesting that continuity and compactness are all that is needed to prove the existence of a continuous and uniformly stabilizing controller.

Remark 2 The continuity of the cost $X$ is counterintuitive in the case where $f$ is sensitively dependent on initial conditions. Intuitively, this continuity means that, although small changes in the initial conditions may lead to drastic changes in the trajectories, the cost to stabilize these trajectories does not change very much. 
Remark 3 Since the LDV controller yields a uniformly exponentially stable LDV system, the LDV controller will locally stabilize the original nonlinear system (3). Hence, if $\|\varphi(0)-\theta(0)\|$ is small enough and the control given by $(7)$ is applied, then $\varphi(k) \rightarrow \theta(k)$ as $k \rightarrow \infty$ where $\varphi(k)$ and $\theta(k)$ are given by (1) and (2) respectively. Furthermore, if the perturbations $w$ and $v$ are small enough and/or infrequent enough, then the system remains stable. See [1] for details.

3 Exploiting Ergodicity to Solve the Functional Algebraic Riccati Equation

(FARE)

Solving any functional equation is a difficult task. Here we take advantage of several properties of complicated dynamics to solve the FARE. These methods are appealing because all that is needed is a transitive trajectory and $A_{\theta}$ and $B_{\theta}$. The function $f$ does not need to be known.

3.1 Solving the FARE on a Transitive Orbit A rather brute force method is based on the finite horizon approximation of the infinite horizon linear quadratic control problem. To this end, one simply defines $X\left(0, N, \theta_{0}\right)$ as the solution to the finite horizon Riccati equation as in (8). It can be shown that $X\left(0, N, \theta_{0}\right) \rightarrow X_{\theta_{0}}$ as $N \rightarrow \infty$, uniformly in $\theta_{o}$. Hence, for $N$ large enough, $X\left(0, N, \theta_{o}\right)$ is a good approximation of $X_{\theta_{0}}$. Furthermore, if $X\left(0, N, \theta_{o}\right)$ is known, then $X\left(0, N-1, f\left(\theta_{o}\right)\right)$ is also known, and if $N-1$ is large enough, then $X\left(0, N-1, f\left(\theta_{0}\right)\right) \approx X_{f_{\left(\theta_{0}\right)}}$. Similarly, we compute $X\left(0, N-k, f^{k}(\theta)\right) \approx X_{f^{k}\left(\theta_{o}\right)}$ for $k \leq K$ where $N-K$ is large. Now, if $\theta_{0}$ is a transitive point, then $E=\left\{f^{k}\left(\theta_{o}\right): 0 \leq k \leq K\right\}$ is a $\varepsilon$-net, and solving $X_{\theta}$ on $E$ provides a good approximation of $X$ everywhere. It is possible to show that this approach will only fail if the LDV system is unstabilizable [1].

\subsection{Solving the FARE on Periodic Orbits}

It is a generic property of diffeomorphisms on a compact set to have a dense set of periodic points [8]. In this case, since the solution to the FARE is continuous, if we solve the FARE on the set of periodic points, then we know the solution everywhere. Similarly, if we solve the FARE on the set of periodic points with period less than $N$, where $N$ is large enough, then we approximately know the solution everywhere. It is possible to solve the FARE on periodic points very efficiently.

Let $\theta$ be a fixed point, i.e., $f(\theta)=\theta$. Then, by equation (6), we have

$X_{\theta}=A_{\theta}^{\prime} X_{\theta} A_{\theta}-A_{\theta}^{\prime} X_{\theta} B_{\theta}\left(I+B_{\theta}^{\prime} X_{\theta} B_{\theta}\right)^{-1} B_{\theta}^{\prime} X_{\theta} A_{\theta}+I$

This can be written more succinctly as $X_{\theta}=\rho_{\theta}\left(X_{\theta}\right)$, where $\rho_{\theta}: \mathbb{R}^{n \times n} \rightarrow \mathbb{R}^{n \times n}$ and depends on $\theta$.
Similarly, if $\theta$ is a periodic point with period $N$, we can write $\rho_{\theta} \circ \rho_{f^{\prime}(\theta)} \circ \cdots \rho_{f^{N-1}(\theta)}\left(X_{\theta}\right)=X_{\theta}$. Hence, $X_{\theta}$ is a fixed point of some map. It turns out that solving the above equation is equivalent to solving the following invariant subspace problem [6],

$$
\left(\prod_{k=0}^{N-1}\left[\begin{array}{ll}
\alpha_{f^{k}(\theta)} & \beta_{f^{k}(\theta)} \\
\gamma_{f^{k}(\theta)} & \delta_{f^{k}(\theta)}
\end{array}\right]\right)\left[\begin{array}{l}
I \\
X_{\theta}
\end{array}\right] \subset\left\{\left[\begin{array}{l}
I \\
X_{\theta}
\end{array}\right]\right\}
$$

where $\left\{\left[\begin{array}{l}I \\ X_{\theta}\end{array}\right]\right\}$ is the span of $\left[\begin{array}{l}I \\ X_{\theta}\end{array}\right]$ and where

$$
\begin{aligned}
\alpha_{\theta} & =A_{\theta}^{-1}, \beta_{\theta}=A_{\theta}^{-1} B_{\theta} B_{\theta}^{\prime}, \\
\gamma_{\theta} & =A_{\theta}^{-1}, \delta_{\theta}=A_{\theta}^{\prime}+A_{\theta}^{-1} B_{\theta} B_{\theta}^{\prime},
\end{aligned}
$$

This invariant subspace problem can be solved very efficiently [9].

As in the case of solving the FARE along a transitive orbit, one can solve the FARE along every periodic orbit if and only if the LDV system is stabilizable [1]

\subsection{Solving the FARE on Recurrent Set}

The above methods do not necessarily lead to computationally efficient methods to solve the FARE. The method based on transitive points has the difficulty that the convergence can be very slow. The method based on periodic points has the drawback that it is not always possible to exactly determine the periodic points. Now a method is presented that solves the FARE along a segment of a recurrent orbit and does not suffer from these drawbacks.

The approach here is to approximate a segment of a recurrent orbit as a periodic orbit. Once this approximately periodic orbit is in hand, one can solve the FARE along this orbit using the efficient methods based on periodic orbits discussed above. To be more specific, define $\rho_{\theta}: \mathbb{R}^{n \times n} \rightarrow \mathbb{R}^{n \times n}$ as above and let $\theta \in R(f)$ where $R(f)$ is the set of recurrent points. Since $\theta$ is recurrent, for $\delta>0$ there exists an $N<\infty$ such that $\left\|\theta-f^{N}(\theta)\right\|<\delta$. Since $X$ is continuous, for $\delta$ small enough, $\left\|X_{\theta}-X_{f^{N}(\theta)}\right\|<\varepsilon$ and

$\left\|\rho_{\theta} \circ \rho_{f(\theta)} \circ \cdots \circ \rho_{f^{N-1}(\theta)}\left(X_{f^{N}(\theta)}\right)-X_{f^{N}(\theta)}\right\|<\varepsilon$.

Therefore, $X_{f^{N}(\theta)}$ is "nearly" the fixed point of

$$
\rho_{\theta} \circ \rho_{f^{1}(\theta)} \circ \cdots \rho_{f^{N-1}(\theta)}(Y)=Y .
$$

In fact,

Theorem 2 Let $\theta$ be a recurrent point and let $\varepsilon>0$. Then there exists a $\delta>0$ and $N<\infty$ such that $\left\|f^{N}(\theta)-\theta\right\|<\delta$ and $\left\|X_{\theta}-Y\right\|<\varepsilon$ where $X$ solves the FARE and $Y$ solves (9).

Remark 4 The proof relies on the Closing Lemma and on the structural stability of the FARE [3]. 


\subsection{Jump Linear Approximation}

There is a strong connection between LDV systems and jump linear systems [7]. Jump linear systems are described by

$$
x^{J L}(k+1)=A_{\sigma(k)}^{J L} x(k)+B_{\sigma(k)}^{J L} u(k)
$$

where $\sigma(k)$ is a Markov chain. It is possible to approximate the LDV system as a jump linear system as follows: Partition $\Theta$ into a finite set of cells $R_{i}$, i.e. $\Theta=\bigcup_{i=1}^{N} R_{i}$. For each cell $R_{i}$ choose a point $r_{i} \in R_{i}$. Define $A_{i}^{J L}=A_{r_{i}}$ and $B_{i}^{J L}=B_{r_{i}}$. Define the transition probabilities $p_{i, j}=\frac{\lambda\left(f\left(R_{i}\right) \cap R_{j}\right)}{\lambda\left(R_{i}\right)}$ where $\lambda$ is Lebesgue measure.

The optimal jump linear controller for a jump linear system is given by $u(k)=F_{\sigma(k)} x(k)$ where

and

$$
F_{\sigma}=-\left(I+B_{\sigma}^{\prime} \hat{Y}_{\sigma} B_{\sigma}\right)^{-1} B_{\sigma}^{\prime} \hat{Y}_{\sigma} A_{\sigma}
$$

$$
\begin{aligned}
Y_{\sigma}= & A_{\sigma}^{\prime} \hat{Y}_{\sigma} A_{\sigma}+I \\
& -A_{\sigma}^{\prime} \hat{Y}_{\sigma} B_{\sigma}\left(I+B_{\sigma}^{\prime} \hat{Y}_{\sigma} B_{\sigma}\right)^{-1} B_{\sigma}^{\prime} \hat{Y}_{\sigma} A_{\sigma}
\end{aligned}
$$

for all $\sigma=1, \cdots, N$ and $\hat{Y}_{\sigma}=\sum_{j=1}^{N} p_{\sigma, j} Y_{j}$. The equations given by (10) form a set of coupled Riccati equations whose solution has been extensively investigated in [5]. In [4] it was shown that as $\max _{i} \operatorname{diam}\left(R_{i}\right) \rightarrow 0$, the solution to the coupled Riccati equations (10) approaches the solution to FARE.

\section{Example}

In the following, an LDV controller is devised for the Hénon system. The Hénon system is defined as $\left[\begin{array}{l}\theta_{1}(k+1) \\ \theta_{2}(k+1)\end{array}\right]=\left[\begin{array}{l}1-(1.4+u(k)) \theta_{1}(k)^{2}+\theta_{2}(k) \\ 0.3 \theta_{1}(k)\end{array}\right]$ with control input $u$. Define $\Theta$ to be the attractor of the uncontrolled Hénon map.

Define

$$
\begin{aligned}
A_{\theta(k)} & =\left.\frac{d f}{d \theta}\right|_{\theta(k), 0}=\left[\begin{array}{cc}
-2 a \theta_{1}(k) & 1 \\
b & 0
\end{array}\right](11) \\
\text { and } B_{\theta(k)} & =\left.\frac{d f}{d u}\right|_{\theta(k), 0}=\left[\begin{array}{c}
-\theta_{1}^{2}(k) \\
0
\end{array}\right] .
\end{aligned}
$$

Since a trajectory that enters every $\varepsilon$-neighborhood for small $\varepsilon$ is easily found, the FARE may be solved using the methods from Section 3.1 or 3.3. In this way the function $F: \Theta \rightarrow \mathbb{R}^{1 \times 2}$ is obtained and the closed-loop tracking error dynamics (as discussed in Section 2) is

$$
\begin{aligned}
& {\left[\begin{array}{l}
x_{1}(k+1) \\
x_{2}(k+1)
\end{array}\right]=} \\
& A_{\theta(k)}+B_{\theta(k)} F_{\theta(k)}\left[\begin{array}{l}
x_{1}(k) \\
\left.x_{2}(k)\right)
\end{array}\right]+\left[\begin{array}{l}
\eta_{1}(k) \\
\eta_{2}(k)
\end{array}\right]
\end{aligned}
$$

where $\eta$ accounts for the error in linearization. Figure 1 shows the feedback gain, $F$, for the LDV system (12). Note that the feedback is continuous on $\Theta$, the attractor of the Hénon map.
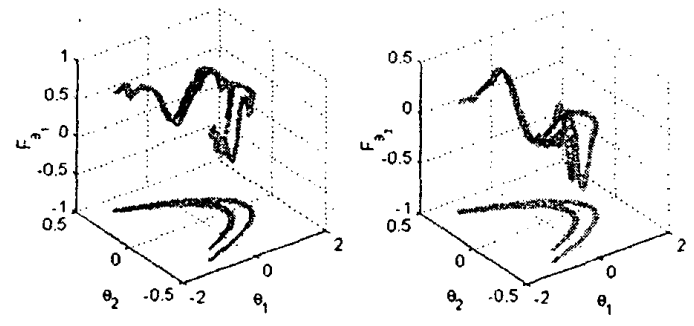

Figure 1: Feedback $F_{\theta}=\left[\begin{array}{ll}F_{\theta_{1}} & F_{\theta_{2}}\end{array}\right]$ for the Hénon map. This shows that the feedback is continuous in $\theta$. A plot of the attractor of the Hénon map in the $\left(\theta_{1}, \theta_{2},-1\right)$ plane is included for reference.

\section{References}

[1] Stephan Bohacek and Edmond Jonckheere. Linear dynamically varying $L Q$ control of systems with complicated dynamics. Submitted to IEEE Transactions on Automatic Control.

[2] Stephan Bohacek and Edmond Jonckheere. Nonlinear tracking over compact sets with linear dynamically varying $H^{\infty}$ control. Siam Journal of Control and Optimization, (Submitted).

[3] Stephan Bohacek and Edmond Jonckheere. Structural stability of LDV controllers. In Conference on Decision and Control, pages 4630-4635, Tampa, Florida, Dec. 16-18, 1998.

[4] Stephan Bohacek and Edmund Jonckheere. Linear dynamically varying (LDV) systems versus jump linear systems. In American Control Conference, pages 4024-4028, San Diego, CA, June 2-4, 1999.

[5] I. Borno and Z. Gajic. Parallel algorithm for solving coupled algebraic Lyapunov equations of discrete-time jump linear systems. Computers Mathematics and Applications, 30:1-4, 1995.

[6] R. Bucy and L. Campbell. Determination of steady state behavior for periodic discrete filtering problem. Computer Mathematics and Applications, 15(2):131-140, 1988.

[7] M. Fragoso, J. Ribeiro DO Val, and D. Pinto. Jump linear $H_{\infty}$ control: The discrete-time case. Control-Theory and Advanced Technology, 10(4):1459-1474, 1995.

[8] Anotole Katok and Boris Hasselblatt. Introduction to the Modern Theory of Dynamical Systems. Cambridge University Press, 1995.

[9] Kemin Zhou, John C. Doyle, and Keith Glover. Robust and Optimal Control. Prentice Hall, 1996. 